\title{
A realidade do consumo de drogas nas populações escolares
}

Adriana Pinheiro,* Paula Picanço,** José Barbeito***

\section{RESUMO}

Objectivos: Caracterizar o consumo de drogas ilícitas dos estudantes açorianos do terceiro ciclo e avaliar os seus conhecimentos sobre a temática.

Tipo de estudo: Observacional, transversal descritivo.

Local: Escolas com ensino de terceiro ciclo da Região Autónoma dos Açores.

População: Estudantes do terceiro ciclo.

Métodos: Aplicação de um inquérito a uma amostra de conveniência, composta pelos alunos do $9 .^{\circ}$ ano de cinco escolas da Região.

Resultados: Foram incluídos no estudo 602 adolescentes, 307 do sexo feminino, com uma média etária de 15,2 (14-18) anos. Todos os inquiridos afirmaram já ter ouvido falar de drogas (76,4\% na escola e $47 \%$ em casa), 62,3\% já viram amigos consumir, a $38 \%$ já foi oferecida droga e $25,6 \%$ já experimentaram (55,8\% dos rapazes e $44,2 \%$ das raparigas). A idade média de início de utilização foi de 14,4 anos, a droga mais utilizada foi a cannabis, o principal local de consumo foi em bar/discoteca mas $41 \%$ já viram consumir na escola e $46,8 \%$ dos consumidores já o fizeram neste local. Quase $43 \%$ dos alunos experimentaram uma ou duas vezes, $44,2 \%$ consomem ao fim-de-semana, $9,7 \%$ duas a três vezes por semana e $3,2 \%$ todos os dias. Os principais motivos de consumo foram a curiosidade e a oferta de um amigo.

Conclusões: Este estudo sugere que a prevalência do consumo de droga entre os adolescentes açorianos é elevada, superior a qualquer outra região do país e à média europeia. Questiona-se a adequação da informação adquirida pelos alunos, tendo em conta que ocorre maioritariamente na escola. De realçar que o consumo ocorreu, sobretudo, em bares e discotecas, abrindo perspectivas de intervenção nas áreas de lazer.

Os resultados alertam para a necessidade de informação e formação adequadas, sensibilizando os jovens para os efeitos nocivos do consumo de estupefacientes e promovendo estilos de vida saudáveis.

Palavras-chave: Drogas Ilícitas; Adolescentes; Comportamentos.

\section{INTRODUÇÃO}

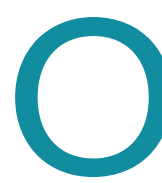

consumo de drogas transformou-se numa preocupação mundial, particularmente nos países industrializados, em função da sua grande prevalência e dos riscos que pode acarretar. A adolescência é uma etapa do desenvolvimento que suscita grandes preocupações quanto ao consumo de drogas pois constitui uma época de exposição e vulnerabilidade às mesmas.

*Assistente de Pediatria do Hospital do Divino Espírito Santo de Ponta Delgada. **Assistente de Medicina Geral e Familiar do Centro de Saúde de Angra do Heroísmo. ***Assistente Graduado de Medicina Geral e Familiar do Centro de Saúde de Angra do Heroísmo.
Os vários Estados têm fomentado o estudo e o controlo do fenómeno do consumo de drogas com o objectivo de definir políticas de intervenção.

O European School Survey on Alcohol and Drugs (ESPAD) é um projecto com inquéritos realizados a cada 4 anos em 35 países europeus, que conta com o apoio do Grupo Pompidou do Conselho da Europa e do Observatório Europeu da Droga e da Toxicodependência (OEDT). O estudo realizado em 2007 concluiu que, em Portugal Continental, $18 \%$ dos rapazes e $10 \%$ das raparigas de 16 anos de idade já experimentaram o consumo de drogas ilícitas pelo menos uma vez. A média europeia de consumo de drogas foi de $23 \%$ no sexo mas- 
culino e $17 \%$ no sexo feminino. ${ }^{1}$

O Inquérito Nacional em Meio Escolar, realizado em 2006, revelou um consumo de cannabis de $10 \%$ dos alunos do $3 .^{\circ}$ ciclo e de $32 \%$ dos alunos do ensino secundário da Região Autónoma dos Açores, superior à prevalência de qualquer outra região do país para os mesmos grupos etários. ${ }^{2}$ Notou-se uma diminuição do consumo em relação ao mesmo estudo em 2001 em todas as regiões, excepto nos alunos açorianos do ensino secundário, em que a prevalência se manteve igual (32\%). ${ }^{3}$

Os objectivos deste estudo foram caracterizar o consumo de drogas ilícitas dos estudantes açorianos do $3 .^{\circ}$ ciclo e avaliar os seus conhecimentos sobre a temática.

\section{MÉTODOS}

Foi realizado um estudo observacional, transversal descritivo no ano de 2009. A população em estudo era constituída pelos estudantes do $3 .^{\circ}$ ciclo da Região Autónoma dos Açores. A amostra foi seleccionada por conveniência sendo composta pelos alunos de todas as turmas do 9. ${ }^{\circ}$ ano de 5 escolas da Região Autónoma dos Açores, o que correspondeu a 605 estudantes, ou seja, $6,4 \%$ do total de alunos inscritos no $3 .^{\circ}$ ciclo na Região nesse ano (9484 alunos). A selecção das escolas dependeu de condicionalismos inerentes à situação geográfica das ilhas. Foi aplicado um inquérito anónimo (Anexo I), após autorização do Conselho Executivo de cada escola e dos pais dos alunos. O questionário foi criado pelos autores, com algumas perguntas adaptadas do ESPAD, ${ }^{1}$ não tendo sido validado. O formulário foi entregue pelos autores no início de uma aula, informando os alunos sobre o objectivo do estudo.

Os inquéritos foram distribuídos explicando-se as instruções para o seu preenchimento e explicitando-se $\mathrm{o}$ anonimato das respostas. Os participantes foram encorajados a responder individualmente, com honestidade e em silêncio. Os alunos foram informados de que poderiam esclarecer qualquer dúvida que surgisse ao longo do preenchimento do inquérito.

Após o seu preenchimento os inquéritos foram dobrados e colocados pelos próprios alunos numa urna fechada e transportados pelos autores, mantendo o anonimato de cada estudante.

As variáveis estudadas foram a idade, sexo, informação sobre drogas e seus efeitos, consumo de drogas, e, em caso afirmativo, local, forma de utilização, tipo de estupefaciente, idade de início, frequência e motivo que levou ao consumo.

Foi utilizado o programa SPSS v. 16.0 na análise estatística.

\section{RESULTADOS}

Foram entregues 605 inquéritos (taxa de resposta de $100 \%$ ), dos quais 3 foram excluídos do estudo por apresentarem respostas incoerentes ou sem fidedignidade (um aluno não respondeu a nenhuma questão, dois responderam seleccionando várias opções para a mesma pergunta). Os questionários incluídos foram respondidos de forma integral.

Assim, a amostra incluiu 602 adolescentes, 307 do sexo feminino e 295 do sexo masculino, com idades compreendidas entre os 14 e os 18 anos. A média de idades foi 15,2 anos, com predomínio da faixa etária dos 14 e 15 anos, 66,7\% dos adolescentes, seguida de 16 e 17 anos, 28,9\% dos estudantes (Quadro I).

Todos os inquiridos afirmaram já ter ouvido falar de drogas e seus efeitos em vários contextos. Cerca de $76,4 \%$ já abordaram o tema na escola e apenas $47,0 \%$ discutiram o problema em casa (Quadro II).

A prevalência de consumo de droga entre os estu-

\begin{tabular}{|lrrr|}
\hline \multicolumn{4}{|c|}{ QUADRO I. Caracterização da amostra } \\
Idade & $\begin{array}{c}\text { Sexo Masculino } \\
(\mathbf{n}=295)\end{array}$ & $\begin{array}{c}\text { Sexo Feminino } \\
(\mathbf{n}=307)\end{array}$ & $\begin{array}{c}\text { Total } \\
(\mathbf{n}=602)\end{array}$ \\
\hline 14 & $69(23,4 \%)$ & $107(34,9 \%)$ & $176(29,2 \%)$ \\
\hline 15 & $116(39,3 \%)$ & $110(35,8 \%)$ & $226(37,5 \%)$ \\
\hline 16 & $65(22,0 \%)$ & $50(16,3 \%)$ & $115(19,1 \%)$ \\
\hline 17 & $34(11,5 \%)$ & $25(8,1 \%)$ & $59(9,8 \%)$ \\
\hline 18 & $11(3,7 \%)$ & $15(4,9 \%)$ & $26(4,3 \%)$ \\
\hline
\end{tabular}

QUADRO II. Local onde os alunos já abordaram o tema das drogas

\begin{tabular}{lc} 
Local de Informação & Total \\
\hline Escola & $460(76,4 \%)$ \\
\hline Casa & $283(47,0 \%)$ \\
\hline Televisão & $425(70,6 \%)$ \\
\hline Amigos & $319(53,0 \%)$
\end{tabular}


dantes foi de $25,6 \%, 29,2 \%$ dos rapazes e $22,1 \%$ das raparigas (Quadro III).

A droga mais utilizada foi a cannabis, já experimentada por todos os indivíduos que afirmaram já ter utilizado drogas ilícitas. A cocaína foi consumida por 5,2\%, o ecstasy também por $5,2 \%$, as anfetaminas por $3,2 \% \mathrm{e}$ a heroína por 1,9\% dos alunos. Em todos os tipos de droga a prevalência de utilização foi sempre superior no sexo masculino (Quadro IV).

Do total de inquiridos, $62,3 \%$ afirmaram já ter visto

\begin{tabular}{|lccc|}
\hline \multicolumn{4}{|l}{ QUADRO III. Prevalência da utilização de drogas ilícitas } \\
$\begin{array}{l}\text { Consumo de } \\
\text { drogas ilícitas }\end{array}$ & $\begin{array}{c}\text { Sexo Masculino } \\
(\mathbf{n}=295)\end{array}$ & $\begin{array}{c}\text { Sexo Feminino } \\
(\mathbf{n}=307)\end{array}$ & $\begin{array}{c}\text { Total } \\
(\mathbf{n}=602)\end{array}$ \\
\hline Sim & $86(29,2 \%)$ & $68(22,1 \%)$ & $154(25,6 \%)$ \\
\hline Não & $209(70,8 \%)$ & $239(77,9 \%)$ & $448(74,4 \%)$ \\
\hline
\end{tabular}

\begin{tabular}{lcrr}
\hline QUADRO IV. Tipo de Droga consumida \\
Tipo de Droga & $\begin{array}{c}\text { Sexo Masculino } \\
(\mathbf{n}=86)\end{array}$ & $\begin{array}{c}\text { Sexo Feminino } \\
(\mathbf{n}=68)\end{array}$ & $\begin{array}{c}\text { Total } \\
(\mathbf{n}=154)\end{array}$ \\
\hline Cannabis & $86(100 \%)$ & $68(100 \%)$ & $154(100 \%)$ \\
\hline Cocaína & $5(5,8 \%)$ & $3(4,4 \%)$ & $8(5,2 \%)$ \\
\hline Ecstasy & $6(7,0 \%)$ & $2(2,9 \%)$ & $8(5,2 \%)$ \\
\hline Anfetaminas & $3(3,5 \%)$ & $2(2,9 \%)$ & $5(3,2 \%)$ \\
\hline Heroína & $2(2,3 \%)$ & $1(1,5 \%)$ & $3(1,9 \%)$
\end{tabular}

amigos ou colegas consumirem drogas ilícitas e 38,0\% afirmaram já lhes ter sido oferecida droga.

A percentagem de consumo de drogas ilícitas entre os estudantes foi semelhante para todos os níveis de escolaridade dos pais (Quadro V).

O Quadro VI mostra os locais de consumo e as vias de administração das drogas. O local de consumo preferencial foi um bar/discoteca, referido por $78,6 \%$ dos alunos que afirmaram já ter consumido drogas. Quanto ao consumo de drogas na escola, $249(41,2 \%)$ dos inquiridos já viram consumir neste local e $72(11,9 \%)$ já o

fizeram, correspondendo a 46,8\% dos alunos que já consumiram droga. Todos estes já experimentaram droga fumada, 8 também a consumiram por via nasal, 13 por via oral e 3 por via endovenosa.

A idade de início de utilização foi em média de 14,4 anos (14,3 para os rapazes e 14,5 para as raparigas), sendo a idade mínima de 10 anos e a máxima de 18 anos (Quadro VII).

Quanto à frequência de consumo, é de notar que $42,9 \%$ correspondeu a uma ou duas experiências na vida, mas $44,2 \%$ já apresentava um consumo regular ao fim-de-semana. Cinco estudantes do sexo masculino já consumiam diariamente, sendo que destes 3 tinham 15 anos, um 16 anos e outro 18 anos (Quadro VIII).

Os motivos de início de consumo (Quadro IX) mais referidos pelos estudantes foram a curiosidade $(80,5 \%)$, a oferta de um amigo $(27,3 \%)$ e a diversão (31,4\% dos rapazes e $17,6 \%$ das raparigas). Estas

\begin{tabular}{|c|c|c|c|c|c|}
\hline \multirow{2}{*}{$\begin{array}{c}\text { Escolaridade do Pai } \\
\text { Total }(n=602)\end{array}$} & \multicolumn{2}{|c|}{ Consumo drogas ilícitas } & \multirow{2}{*}{$\begin{array}{c}\text { Escolaridade da mãe } \\
\text { Total }(n=602)\end{array}$} & \multicolumn{2}{|c|}{ Consumo drogas ilícitas } \\
\hline & $\operatorname{Sim}(n=154)$ & Não $(n=448)$ & & $\operatorname{Sim}(n=154)$ & Não $(n=448)$ \\
\hline Sem estudo $(n=15)$ & $6(40,0 \%)$ & $9(60,0 \%)$ & Sem estudo $(n=3)$ & $1(33,3 \%)$ & $2(66,7 \%)$ \\
\hline $1-4 .^{\circ}$ ano $(n=237)$ & $71(30,0 \%)$ & $166(70,0 \%)$ & $1-4 .^{\circ}$ ano $(n=239)$ & $73(30,5 \%)$ & $166(69,5 \%)$ \\
\hline 5-6..$^{\circ}$ ano $(n=104)$ & $24(23,1 \%)$ & $80(76,9 \%)$ & $5-6 .^{\circ}$ ano $(n=105)$ & $28(26,7 \%)$ & $77(73,3 \%)$ \\
\hline $7-9 .^{\circ}$ ano $(n=97)$ & $20(20,6 \%)$ & $77(79,4 \%)$ & $7-9 .^{\circ}$ ano $(n=94)$ & $21(22,3 \%)$ & $73(77,7 \%)$ \\
\hline $10-12 .^{\circ}$ ano $(n=102)$ & $22(21,6 \%)$ & $80(78,4 \%)$ & $10-12 .^{\circ}$ ano $(n=93)$ & $16(17,2 \%)$ & $77(82,8 \%)$ \\
\hline Curso superior $(n=38)$ & $8(21,1 \%)$ & $30(78,9 \%)$ & Curso superior $(n=60)$ & $12(20,0 \%)$ & $48(80,0 \%)$ \\
\hline Desconhece/NR $(n=9)$ & $3(33,3 \%)$ & $6(66,7 \%)$ & Desconhece/NR $(n=8)$ & $2(25,0 \%)$ & $6(75,0 \%)$ \\
\hline
\end{tabular}


razões foram escolhidas pelos estudantes a partir de sete opções, incluindo «outras». Vários motivos podiam ser apontados. Dois estudantes afirmaram ter consumido em momentos de solidão.

\section{DISCUSSÃO}

Este estudo mostra uma prevalência de consumo de drogas ilícitas de $25,6 \%$ (29,2\% nos rapazes e $22,1 \%$ nas raparigas) nos alunos respondedores. Estes valores são

\begin{tabular}{|c|c|c|c|}
\hline & $\begin{array}{l}\text { Sexo Masculino } \\
\qquad(n=86)\end{array}$ & $\begin{array}{l}\text { Sexo Feminino } \\
\qquad(n=68)\end{array}$ & $\begin{array}{c}\text { Total } \\
(n=154)\end{array}$ \\
\hline \multicolumn{4}{|l|}{ Local de Consumo } \\
\hline Bar/discoteca & $64(74,4 \%)$ & $57(83,8 \%)$ & $121(78,6 \%)$ \\
\hline Escola & $47(54,7 \%)$ & $25(36,8 \%)$ & $72(46,8 \%)$ \\
\hline Casa & $14(16,3 \%)$ & $6(8,8 \%)$ & $20(13,0 \%)$ \\
\hline \multicolumn{4}{|c|}{ Via de administração } \\
\hline Fumada & $86(100 \%)$ & $68(100 \%)$ & $154(100 \%)$ \\
\hline Inalada & $5(5,8 \%)$ & $3(4,4 \%)$ & $8(5,2 \%)$ \\
\hline Em comprimidos & $9(10,5 \%)$ & $4(5,9 \%)$ & $13(8,4 \%)$ \\
\hline Injectada & $2(2,3 \%)$ & $1(1,5 \%)$ & $3(1,9 \%)$ \\
\hline
\end{tabular}

superiores à média europeia de consumidores, identificada em estudos internacionais. A média de adolescentes entre os 15 e os 16 anos que já experimentaram drogas ilícitas pelo menos uma vez na vida, dos países europeus participantes no ESPAD, situou-se nos $23 \%$ nos rapazes e $17 \%$ nas raparigas, com variação considerável entre os vários países. Os níveis de prevalência mais reduzidos encontraram-se nos países nórdicos e Europa Oriental. Na República Checa, $46 \%$ dos adolescentes referiram ter consumido drogas ilícitas. Na Suíça, França e República Eslovaca cerca de $30 \%$. Em Portugal 13\%. Na Noruega, Chipre e Roménia apenas $6 \%{ }^{1}$

A prevalência de consumo de drogas entre os estudantes encontrada nesta amostra é superior à prevalência encontrada em qualquer outra região do país num estudo de 2006 em estudantes do $3 .{ }^{\circ}$ ciclo (Norte $6 \%$, Centro $6 \%$, Lisboa-Vale do Tejo 6\%, Alentejo 8\%, Algarve $7 \%$ e Madeira 7\%). Nos Açores a prevalência encontrada no referido estudo foi de $10 \%{ }^{2}$

Tal como noutros estudos, são os rapazes quem mais frequentemente consome. ${ }^{4,5}$

A idade de início de consumo foi em média de 14,4 anos, semelhante à encontrada por outros autores. ${ }^{6-8}$

\section{QUADRO VII. Idade de início de consumo de drogas}

\begin{tabular}{lccr}
$\begin{array}{l}\text { Idade de Início } \\
\text { (anos) }\end{array}$ & $\begin{array}{c}\text { Sexo masculino } \\
(\mathbf{n}=\mathbf{8 6})\end{array}$ & $\begin{array}{c}\text { Sexo feminino } \\
(\mathbf{n}=68)\end{array}$ & $\begin{array}{c}\text { Total } \\
(\mathbf{n}=154)\end{array}$ \\
\hline Média & 14,3 & 14,5 & 14,4 \\
\hline Mediana & 14,0 & 14,0 & 14,0 \\
\hline Desvio-padrão & 1,5 & 1,3 & 1,4 \\
\hline Mínimo-Máximo & $10-17$ & $10-18$ & $10-18$ \\
\hline
\end{tabular}

\section{QUADRO VIII. Frequência de consumo}

\begin{tabular}{lrrr} 
Frequência de consumo & $\begin{array}{c}\text { Sexo Masculino } \\
(\mathbf{n}=86)\end{array}$ & $\begin{array}{c}\text { Sexo Feminino } \\
(\mathbf{n}=68)\end{array}$ & $\begin{array}{c}\text { Total } \\
(\mathbf{n}=154)\end{array}$ \\
\hline Experimentou 1 ou 2 vezes & $34(39,5 \%)$ & $32(47,1 \%)$ & $66(42,9 \%)$ \\
\hline Fim-de-semana & $38(44,2 \%)$ & $30(44,1 \%)$ & $68(44,2 \%)$ \\
\hline 2-3 vezes/semana & $9(10,5 \%)$ & $6(8,8 \%)$ & $15(9,7 \%)$ \\
\hline Todos os dias & $5(5,8 \%)$ & $0(0,0 \%)$ & $5(3,2 \%)$
\end{tabular}

A droga mais consumida foi a cannabis, utilizada por todos os estudantes que afirmaram já ter experimentado algum tipo de droga ilícita. Este resultado é semelhante ao encontrado noutros trabalhos. ${ }^{6,9}$

No presente estudo, 19 alunos (3,2\%) tinham já experimentado uma ou mais drogas além da cannabis. A média europeia de utilização de outras drogas situou-se em 7\% em 2007. ${ }^{1}$

Vários são os factores de risco que têm sido implicados no consumo de drogas ilícitas, tais como factores genéticos, neurobiológicos, de personalidade e psicológicos. Contudo, o meio familiar e o ambiente em que o adolescente se insere são considerados factores de risco major para o abuso de substâncias lícitas e ilícitas. ${ }^{10-13}$

As relações positivas na família, o suporte emocional e social dos pais e um estilo de disciplina parental construtivo e consistente tendem a estar relaciona- 


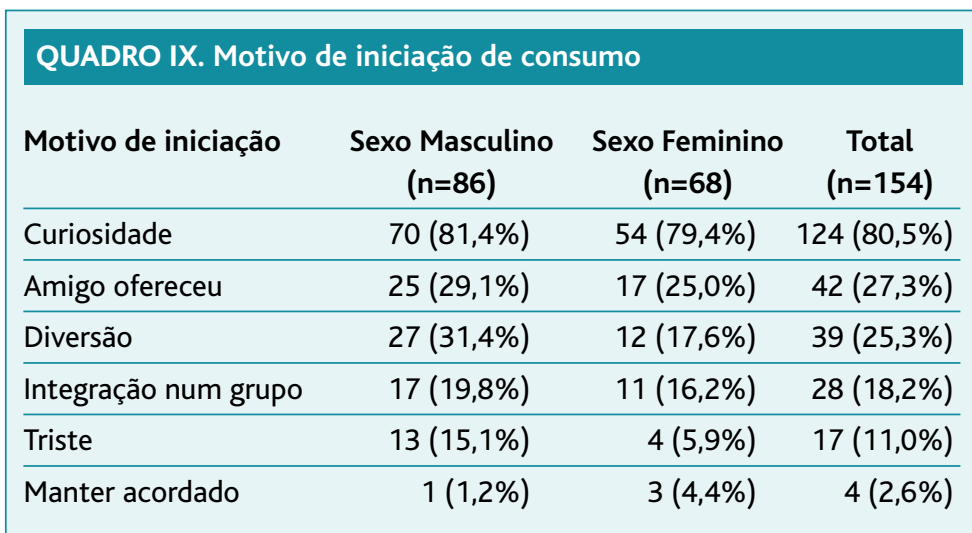

dos com menos envolvimento em comportamentos de risco. ${ }^{14,15}$

O facto de os jovens passarem o tempo livre em contextos sociais não estruturados está relacionado com um maior uso de substâncias. ${ }^{16}$ Ao contrário, a participação em actividades organizadas funciona como um factor de protecção. ${ }^{17}$

Existem vários tipos de consumo de drogas, nomeadamente um consumo tipo festivo, que acontece em grupo e através do qual se procura divertimento, ${ }^{18} \mathrm{e} \mathrm{um}$ consumo autoterapêutico que procura o alívio de sintomas de mal-estar e stress. ${ }^{18-20}$ Os principais locais de consumo foram realmente os locais de lazer, nomeadamente bares e discotecas (78,6\%). Quanto aos factores motivadores do consumo, o desejo de diversão foi referido por $25,3 \%$ e o facto de estar triste por $8,4 \%$ dos participantes.

A curiosidade foi o factor motivante da experimentação referido por mais adolescentes $(45,5 \%)$, facto de acordo com outros estudos realizados noutros países. ${ }^{7,8,21}$

Neste estudo verificou-se uma percentagem elevada de alunos que consome droga em ambiente escolar. Do total de inquiridos, $41,2 \%$ já viram consumir na escola e $46,7 \%$ dos consumidores já o fizeram neste local. Isto revelou-se um factor extremamente inquietante, uma vez que a escola deveria ser tida como um local privilegiado para prevenir estes comportamentos de risco. No entanto, é o local de convívio entre pares e em que essas condutas se perpetuam, de certo modo como tentativa de afirmação. A intervenção na escola, pelo que foi possível apurar, tem sido feita sobretudo através de sessões de esclarecimento, organizadas pelos
Centros de Saúde e que envolvem médicos de Medicina Geral e Familiar, enfermeiros e psicólogos. Contudo, as intervenções realizadas, apesar de fundamentais, parecem não ser suficientes.

Dependendo de vários factores, a escola pode ser um factor protector ou propiciador de comportamentos de risco. ${ }^{22}$ Um ambiente escolar que seja promotor de saúde pode ser encarado como um recurso para o desenvolvimento de comportamentos de aumento de saúde e de bem-estar, sendo que a satisfação com a escola pode contribuir para esse bem-estar e para a qualidade de vida dos jovens. O contexto escolar (grupo de pares) e social em que o adolescente está inserido tem sido apontado como o preditor mais consistente do uso de substâncias na adolescência. ${ }^{23}$ No seio do ambiente escolar identificam-se factores como a falta de motivação para a aprendizagem, o fraco desempenho escolar, o absentismo e a vontade de ser independente, articulados com a falta de interesse na realização pessoal como factores de risco para o uso de substâncias ilícitas. ${ }^{24}$ Por outro lado, a atitude dos amigos em relação à escola parece ser um factor determinante na forma como os adolescentes vêem a escola. ${ }^{25,26}$ Neste estudo, o desejo de integração num grupo foi referido como motivador do consumo por $18,2 \%$ dos utilizadores, sendo que a experimentação levada pela oferta de um amigo foi referida por $16,2 \%$.

A disponibilidade da droga parece ser um factor importante no consumo. Cerca de $62,3 \%$ dos estudantes afirmaram já ter visto amigos ou colegas consumirem drogas ilícitas e 38,0\% afirmaram já lhes ter sido oferecida droga. A nível europeu, cerca de 30,0\% dos estudantes referiram ser fácil encontrar cannabis. ${ }^{1}$

As intervenções com o intuito de prevenir o consumo de substâncias psico-activas a nível escolar são fundamentais, havendo necessidade de uma abordagem dos múltiplos problemas do contexto escolar, tal como tem sido proposto e legislado recentemente. ${ }^{2}$ Estes resultados também alertam para a necessidade de se intervir em factores específicos associados ao risco, como os locais de diversão frequentados pelos adolescentes. Contudo, a socialização com o grupo de pares é fundamental no desenvolvimento, bem-estar e auto-estima 
dos jovens, pelo que as intervenções têm que abordar alternativas para lidar com os desafios com que os adolescentes se deparam, permitindo-lhes alcançar estabilidade pessoal e social, sem recorrer ao uso de drogas.

Em conclusão, este estudo sugere que a prevalência de consumo de droga entre os adolescentes açorianos é muito elevada, superior a qualquer outra região do país e à média europeia.

No entanto, é importante realçar as limitações metodológicas deste estudo, que poderão ter contribuído para este valor tão elevado. O facto de se terem seleccionado apenas os alunos do $9 .^{\circ}$ ano, os seja, os mais velhos do $3 .^{\circ}$ ciclo, pode ter influenciado a elevada percentagem de estudantes que referiram já ter consumido substâncias ilícitas, quando comparada com outros estudos. Também a utilização de uma amostra de conveniência, muito provavelmente não representativa da população em estudo, impede a generalização dos resultados para a população estudantil do $3 .^{\circ}$ ciclo, como era o objectivo do trabalho. Por outro lado, como o estudo foi realizado apenas em ambiente escolar não é possível generalizar estes resultados para a população adolescente da Região Autónoma dos Açores.

A não validação do questionário é uma limitação importante, tanto mais que se admite que algumas perguntas podem ter induzido as respostas.

\section{REFERÊNCIAS BIBLIOGRÁFICAS}

1. Hibell B, Guttormsson U, Ahlström S, Balakireva O, Bjarnason T, Kokkevi A, et al. The 2007 ESPAD Report: substance use among students in 35 European countries. Stockholm: The Swedish Council for Information on Alcohol and other Drugs (CAN), The European Monitoring Centre for Drugs and Drug Addiction (EMCDDA) and Council of Europe, Cooperation Group to Combat Drug Abuse and Illicit Trafficking in Drugs (Pompidou Group); 2009. Disponível em: http://www.espad.org/espadreports [acedido em 26/07/2010].

2. Feijão F. Inquérito Nacional em Meio Escolar - 2006. Consumo de drogas e outras substâncias psicoactivas: uma abordagem integrada. IDT 2008. Acessível em http://www.idt.pt/pt/investigacao/paginas/estudosconcluidos.aspx [acedido em 26/07/2010].

3. Feijão F. Inquérito Nacional em Meio Escolar - 2001. Consumo de drogas e outras substâncias psicoactivas: uma abordagem integrada. IDT 2004. Disponível em: http://www.idt.pt/pt/investigacao/paginas/estudosconcluidos.aspx [acedido em 26/07/2010].

4. Kokkevi A, Richardson C, Florescu S, Kuzman M, Stergar E. Psychosocial correlates of substance use in adolescence: a cross-national study in six european countries. Drug Alcohol Depend 2007 Jan 5; 86 (1): 6774.
5. Naia A, Simões C, Matos M. Consumo de substâncias na adolescência. Toxicodependências 2007 Sep; 13 (3): 23-30.

6. Madu SN, Matla MQ. Illicit drug use, cigarette smoking and alcohol drinking behaviour among a sample of high school adolescents in the Pietersburg area of the Northern Province, South Africa. J Adolesc 2003 Feb;26 (1): 121-36.

7. Saluja BS, Grover S, Irpati AS, Mattoo SK, Basu D. Drug dependence in adolescents 1978-2003: a clinical-based observation from North India. Indian J Pediatr 2007 May; 74 (5): 455-8.

8. Veress K, Wheeler JG, Ramsay M, McMichael AJ. Drug-use patterns and risk factors among young offenders in Hungary: an epidemiological study. Int J Drug Policy 2004 Sep; 15 (4): 285-95.

9. Webb E, Ashton Ch, Kelly P, Kamali F. Alcohol and drug use in UK university students. Lancet 1996 Oct 5; 348 (9032): 922-5.

10. Fishman $M$, Bruner $A$, Adger $H$ Jr. Substance abuse among children and adolescents. Pediatr Rev 1997 Nov; 18 (11): 394-403.

11. Allison KW, Crawford I, Leone PE, Trickett E, Perez-Febles A, Burton LM, et al.Adolescent substance use: preliminary examinations of school and neighbourhood context. Am J Community Psychol 1999 Apr; 27 (2): 111-41.

12. Sneed CD, Morisky DE, Rotheram-Borus MJ, Ebin VJ, Malotte CK. Patterns of adolescent alcohol, cigarette, and marijuana use over a 6month period. Addict Behav 2001 May-Jun; 26 (3): 415-23.

13. Wiesner M, Ittel A. Relations of pubertal timing and depressive symptoms to substance use IN early adolescence. J Early Adolesc 2002 Feb; 22 (1): 5-23.

14. Ardelt M, Day L. Parents, siblings and peers: close social relationships and adolescent deviance. J Early Adolesc 2002 Aug; 22 (3): 310-49.

15. Mounts NS. Parental management of adolescent peer relationships in context: the role of parenting style. J Fam Psychol 2002 Jul; 16 (1): 5889.

16. Caldwell LL, Darling N. Leisure context, parental control and resistance to peer pressure as predictors of adolescent partying and substance abuse: an ecological perspective. J Leisure Res 1999 Jan; 31 (1): 1-24.

17. Darling N, Caldwell LL, Smith R. Participation in school-based extracurricular activities and adolescent adjustment. J Leisure Res 2005 Jan; 37 (1): 1-41.

18. Braconnier, A, Marcelli D. As mil faces da adolescência. Lisboa: CLIMEPSI Editores; 2000.

19. Simantov E, Schoen C, Klein J. Health compromising behaviors: why adolescents smoke or drink? Identifying underlying risk and protective factors. Arch Pediatr Adolesc Med 2000 Oct; 154 (10): 1025-33.

20. Whalen CK, Jamner LD, Henker B, Delfino RJ. Smoking and moods in adolescents with depressive and aggressive dispositions: evidence from surveys and electronic diaries. Health Psychol 2001 Mar; 20 (2): $99-$ 111.

21. Spear LP. The adolescent brain and age-related behavioral manifestations. Neurosci Biobehav Rev 2000 Jun; 24 (4): 417-63.

22. Zaff JF, Moore KA, Papillo AR, Williams S. Implications of extracurricular activity participation during adolescence on positive outcomes. J Adolesc Res 2003 Nov; 18 (6): 599-630.

23. Kuntsche E, Jordan MD. Adolescent alcohol and cannabis use in relation to peer and school factors: results of multilevel analyses. Drug Alcohol Depend 2006 Sep; 84 (2): 167-74. 
24. Sutherland I, Shepherd JP. Social dimensions of adolescent substance use. Addiction 2001 Mar; 96 (3): 445-58.

25. Akers JF, Jones RM, Coyl DD. Adolescent friendship pairs: similarities in identity status development, behaviors, attitudes, and intentions. J Adolesc Res 1998 Apr; 13 (2): 178-201.

26. Ryan AM. The peer group as a context for the development of young adolescent motivation and achievement. Child Dev 2001 Jul-Aug; 72 (4): 1135-50.

27. Sousa A, Pinto A, Sampaio D, Nunes E, Baptista M, Marques P. Consumo de Substâncias Psicoactivas e Prevenção em Meio Escolar. Editorial do Ministério da Educação. 2007 Sep. Disponível em: http://sitio.dgidc.minedu.pt/saude/documents/consumo_prev.pdf [acedido em 26/07/2010].

\section{CONFLITOS DE INTERESSE}

Os autores declaram não possuir qualquer tipo de conflitos de interesse.

\section{ENDEREÇO PARA CORRESPONDÊNCIA}

Adriana Paula Pontes Pinheiro de Castro

Rua Capitão Borges Pamplona, 8

9760-431 Praia da Vitória

E-mail: adrianappp@hotmail.com

Recebido em 27/07/2010

Aceite para publicação em 28/07/2011

\section{ABSTRACT}

\section{THE REALITY OF DRUG USE IN HIGH SCHOOLS IN THE AZORES}

Objectives: To characterize illegal drug use among Azorean high school students and to assess their knowledge of this subject. Type of study: Cross-sectional.

Setting: High schools in the Autonomous Region of the Azores.

Population: High school students in the ninth grade

Methods: A survey was conducted in a convenience sample of ninth grade students in five high schools in the region.

Results: The study included 602 teenagers, of who 307 were females, with an average age of 15.2 (14-18) years. All respondents said they had already heard of drugs ( $76.4 \%$ at school and $47 \%$ at home), $62.3 \%$ have seen friends consume drugs, $38.0 \%$ had been offered drugs and $25.6 \%$ had tried drugs ( $55.8 \%$ of the boys and $44.2 \%$ of the girls). The average age of first use was 14.4 years and the most commonly drug used was cannabis. The main place of consumption was in bar or nightclub but $41 \%$ had seen drug use at school and $46.8 \%$ of users have done on this at school. Almost $43 \%$ of students had experienced drug use once or twice, $44.2 \%$ used on weekends, $9.7 \%$ used two to three times a week and 3.2\% used drugs every day. The main reasons for drug use were curiosity and the offer of a friend.

Conclusions: This study suggests that the prevalence of drug use among Azorean teenagers is high, more than any other region of the country, and higher than the European average. We question the adequacy of information on drug use obtained by the students, considering that this occurs mainly in school. Drug use occurred most often in bars and nightclubs, suggesting possibilities for intervention in these areas. These results suggest the need for adequate information and training, raising awareness of young people to the harmful effects of drug abuse, and promoting healthy lifestyles.

Keywords: Illegal Drugs; Teenagers; Behaviours. 


\section{ANEXO I \\ INQUÉRITO}

Este é um inquérito anónimo. Não escrevas qualquer elemento que te identifique.

Por favor, responde com o máximo de sinceridade.

Responde a todas as questões.

As perguntas $4,7,9,10,11$ e 14 podem ter mais do que uma resposta, se achares que é o que mais se adequa à tua situação.

Quando acabares de responder, dobra o inquérito e coloca-o na caixa que se encontra na sala para o efeito.

1. Idade

2. Sexo

$M$

$\mathrm{F}$

3. Escolaridade do pai:

Sem estudo

$1-4 .^{\circ}$ anos

5-6. anos

7-9. anos

10-12. anos

Formação superior

Não sei

Escolaridade da mãe:

Sem estudo

$1-4 .^{\circ}$ anos

5-6. anos

7-9. anos

10-12. anos

Formação superior

Não sei

4. Certamente já ouviste falar dos vários tipos de droga e dos seus efeitos.

Onde ouviste?

Em casa

$\mathrm{Na}$ escola

$\mathrm{Na}$ televisão

Com os amigos

Noutro local

Nunca ouvi

5. Algum amigo/colega já te ofereceu alguma droga para experimentares?

Sim Não

6. Já viste os teus colegas/amigos usarem drogas?

Sim

Não

7. Onde viste?

$\mathrm{Na}$ escola

Num bar/discoteca

Em casa deles

Nunca vi
8. Já experimentaste alguma droga?

Sim

Não

9. Onde experimentaste?

$\mathrm{Na}$ escola

Num bar/discoteca

Em casa

Nunca experimentei

10. De que forma experimentaste?

Fumada

Snifada

Injectada

Em comprimidos

Nunca experimentei

11. Qual é que experimentaste?

Haxixe (chamon, pedra, charro, ganza, erva)

Extasy (roda, rodinhas, pastilhas, pombas)

Heroína (heroa, burra, castanha, bolha)

Cocaína (coca, branca, traço/linha de coca)

Anfetaminas (speeds)

"Drunfos"

Nenhuma

12. Que idade tinhas quando experimentaste a primeira vez?

13. Qual a frequência com que usas as drogas?

Usei 1 ou 2 vezes na vida

Só ao fim-de-semana

2-3x/semana

Todos os dias

Nunca

14. Começaste a usar as drogas:

Por curiosidade

Porque um amigo me ofereceu

Porque estava triste

Porque os meus amigos usam e também quis experimentar

Para me manter acordado

Para me divertir

Para me integrar num grupo

Outra razão.

Qual?

Nunca usei 